\title{
Comprehensive transcriptome analysis reveals genes in response to water deficit in the leaves of Saccharum narenga (Nees ex Steud.) hack
}

Xihui Liu ${ }^{1,2+}$ D, Ronghua Zhang ${ }^{1 \dagger}$, Huiping Ou ${ }^{1 \dagger}$, Yiyun Gui ${ }^{1}$, Jinju Wei ${ }^{1}$, Hui Zhou ${ }^{1}$, Hongwei $\operatorname{Tan}^{1 *}$ and Yangrui $\mathrm{Li}^{1,2^{*}}$

\begin{abstract}
Background: Sugarcane is an important sugar and energy crop that is widely planted in the world. Among the environmental stresses, the water-deficit stress is the most limiting to plant productivity. Some groups have used PCR-based and microarray technologies to investigate the gene expression changes of multiple sugarcane cultivars under water stress. Our knowledge about sugarcane genes in response to water deficit is still poor.

Results: A wild sugarcane type, Saccharum narenga, was selected and treated with drought stress for 22 days. Leaves from drought treated (DTS) and control (CK) plants were obtained for deep sequencing. Paired-end sequencing enabled us to assemble 104,644 genes (N50 = 1605 bp), of which 38,721 were aligned to other databases, such as UniProt, NR, GO, KEGG and Pfam. Single-end and paired-end sequencing identified 30,297 genes (> 5 TPM) in all samples. Compared to CK, 3389 differentially expressed genes (DEGs) were identified in DTS samples, comprising 1772 up-regulated and 1617 down-regulated genes. Functional analysis showed that the DEGs were involved in biological pathways like response to blue light, metabolic pathways and plant hormone signal transduction. We further observed the expression patterns of several important gene families, including aquaporins, late embryogenesis abundant proteins, auxin related proteins, transcription factors (TFs), heat shock proteins, light harvesting chlorophyll a-b binding proteins, disease resistance proteins, and ribosomal proteins. Interestingly, the regulation of genes varied among different subfamilies of aquaporin and ribosomal proteins. In addition, DIVARICATA and heat stress TFs were first reported in sugarcane leaves in response to water deficit. Further, we showed potential miRNAs that might be involved in the regulation of gene changes in sugarcane leaves under the water-deficit stress.

Conclusions: This is the first transcriptome study of Saccharum narenga and the assembled genes are a valuable resource for future research. Our findings will improve the understanding of the mechanism of gene regulation in sugarcane leaves under the water-deficit stress. The output of this study will also contribute to the sugarcane breeding program.
\end{abstract}

Keywords: Water deficit, Drought, Sugarcane, Leaves, Transcriptome

\footnotetext{
*Correspondence: hwtan@gxaas.net; liyr@gxaas.net

${ }^{+}$Xihui Liu, Ronghua Zhang and Huiping Ou contributed equally to this work.

'Key Laboratory of Sugarcane Biotechnology and Genetic Improvement (Guangxi), Guangxi Key Laboratory of Sugarcane Genetic Improvement, Ministry of Agriculture, Sugarcane Research Center, Chinese Academy of Agricultural Sciences, Sugarcane Research Institute, Guangxi Academy of Agricultural Sciences, Nanning 530007, China

Full list of author information is available at the end of the article
}

(c) The Author(s). 2018 Open Access This article is distributed under the terms of the Creative Commons Attribution 4.0 International License (http://creativecommons.org/licenses/by/4.0/), which permits unrestricted use, distribution, and reproduction in any medium, provided you give appropriate credit to the original author(s) and the source, provide a link to the Creative Commons license, and indicate if changes were made. The Creative Commons Public Domain Dedication waiver (http://creativecommons.org/publicdomain/zero/1.0/) applies to the data made available in this article, unless otherwise stated. 


\section{Background}

Sugarcane is a $\mathrm{C}_{4}$ grass that belongs to the family Poaceae, sub-family Panicoideae and tribe Andropogoneae. It is an important industrial crop widely grown in tropical and subtropical areas, due to the highly yield of sugar [1]. However, the production of sugarcane is always affected by the environmental stresses, such as water, cold and salt. Among these conditions water stress is the most limiting to plant productivity [1].

Water stress always induces a number of biochemical and physiological responses in plants, such as stomatal closure, repression of cell growth and photosynthesis, and activation of respiration [2]. Studies have been demonstrated to understand the gene expression changes under the water-deficit stress in several model species, such as Arabidopsis [3, 4], maize [5], rice [6], tomato [7], banana [8], soybean [9], and other plants [10-13]. They have shown that abscisic acid (ABA)-dependent and ABA-independent regulatory systems are two major pathways for plants to defense against the water-deficit stress [14]. According to their functions, genes in response to water deficit can be divided into two groups. The first group of gene products are involved in the protection of cells and in the regulation of signal transduction pathways of stress responses, such as chaperons, late embryogenesis abundant (LEA) proteins, water channel proteins, heat shock proteins and lipid-transfer proteins. The second group of gene products in response to water deficit in plants is comprised of regulatory proteins that are involved in further regulation of signal transduction and stress-responsive gene expression, such as various TFs and dehydration-responsive elements $[2,14,15]$.

Some studies have used PCR and microarray technologies to investigate the gene expression profiles of different sugarcane cultivars under water stress. For example, Rodrigues and colleagues identified 91 genes up-regulated in both tolerant (SP83-5073) and sensitive (SP90-1638) sugarcane cultivars, such as heat shock protein 17.2, resistance protein LR10 and transcription factor E2Fe [1]. Carolina and colleagues identified 928 sense transcripts and 59 antisense transcripts differentially expressed in the aerial parts of sugarcane (SP90-1638) submitted to drought for 24, 72 and 120 h [16]. Another study by Rodrigues and colleagues has shown that 1670 genes were differentially expressed in sugarcane (SP83-2847) under mild, moderate and severe water deficit stresses [17]. Gupta and colleagues have identified 25 clusters (EST groups) induced by water-deficit stress in sugarcane (CoS 767) [18]. Iskandar and colleagues have tested 51 genes in the culms of multiple sugarcane cultivars and reported water-deficit stress related genes including genes encoding enzymes involved in amino acid metabolism, a sugar transporter and a transcription factor [11]. Prabu and colleagues have investigated the gene expression profiles of sugarcane (Co740) under varied levels of water deficiency stress using PCR-based cDNA suppression subtractive hybridization technique and have found 158 clones up-regulated under water-deficit stress, which mainly function in cellular organization, protein metabolism, signal transduction, and transcription [19]. However, our knowledge of the gene expression profiles and gene regulation in sugarcane leaves under the water-deficit stress is still poor.

Saccharum narenga (Nees ex Steud.) Hack, also named as Narenga porphyrocoma (Hance) Bor, is a wild species of sugarcane distributed mainly in Asia-temperate (China and eastern Asia) and Asia-tropical (India and Indo-China) areas. This species has a smaller genome size $(2 n=30)$ and many excellent characters, such as drought tolerance, precocity, stocky stem, high tillering ability, red rot tolerance, smut tolerance and mosaic disease resistance. These advantages enable it to be used in sugarcane breeding program. To understand the gene expression profiles of this species under the water-deficit stress, both paired-end and single-end sequencing technologies were used to sequence the cDNA libraries of drought treated leaves and control (CK) plants. Differential expression analysis identified some gene products that have been reported to be involved in response to water deficit, such as various transcription factors (TFs), dehydrins, aquaporins, heat shock proteins, ribosomal proteins and auxin-related proteins. We not only identified novel water-deficit related genes in sugarcane leaves, such as light harvesting chlorophyll a-b binding protein and multiple TFs, but also found, for the first time, that the regulation of genes differ across aquaporin and ribosomal protein subfamilies. Our results will contribute to understand the drought tolerance mechanism in sugarcane and will contribute to the field of sugarcane breeding program. This is the first transcriptome of Saccharum narenga species, so the gene sequences can be referred by future studies.

\section{Methods \\ Plant material and drought treatment}

The Saccharum narenga plants were collected from a barren mountain not far from Nanning of China (22 $53^{\prime}$ $\left.06.7^{\prime \prime} \mathrm{N} 108^{\circ} 21^{\prime} 36.6^{\prime \prime} \mathrm{E}\right)$. They were proved to be Guangxi Hebawang NO. 1, which is from the clonal Saccharum narenga. Six sugarcane plantlets were transplanted in a pod filled with a mixture of peat soil, washed sand, vermiculite and perlite (total weight: $\sim 17.5 \mathrm{~kg}$ ). Then, $\sim 20$ pods were transferred and maintained in a greenhouse of Guangxi Academy of Agricultural Sciences on 2nd May 2014. The greenhouse is equipped with a system for monitoring temperature and relative humidity (mean temperature: $29^{\circ} \pm 4{ }^{\circ} \mathrm{C}$, mean relative humidity: $\left.75 \pm 5 \%\right)$. Every day the plants were watered sufficiently $(\sim 800 \mathrm{~mL}$ per pod). After 6 months, three pods (DTS-R1, DTS-R2 
and DTS-R3) were randomly selected for drought treatment while another three (CK-R1, CK-R2 and CK-R3) pods which have similar growing plants were used as CK. Drought treatment was performed by withholding water for 22 days. One leaf from each pod was randomly selected, immediately stored in liquid nitrogen and stored at $-80^{\circ} \mathrm{C}$ before RNA isolation.

\section{Total RNA extraction}

Total RNA of each leaf was extracted by using TRIzol reagent, as previously described [20,21]. In brief, $100 \mathrm{mg}$ of leaf sample were mixed with $1 \mathrm{~mL}$ TRIzol reagent, homogenized using a power homogenizer and centrifuged at $12000 \times \mathrm{g}$ for $10 \mathrm{~min}$ at $4{ }^{\circ} \mathrm{C}$. Then, the fatty layer was removed and discarded. The supernatant was transferred into a new tube and added with chloroform $(0.2 \mathrm{~mL})$. After shaking for $15 \mathrm{~s}$, the tube was incubated at room temperature for $3 \mathrm{~min}$ and centrifuged at $12000 \times \mathrm{g}$ for 15 min at $4{ }^{\circ} \mathrm{C}$. The aqueous phase was moved into a new tube and added with RNase-free glycogen $(10 \mu \mathrm{g})$ and $100 \%$ isopropanol $(0.5 \mathrm{~mL})$, followed by an incubation at room temperature for $10 \mathrm{~min}$. Then, the tube was centrifuged at $12000 \times \mathrm{g}$ for $10 \mathrm{~min}$ at $4{ }^{\circ} \mathrm{C}$ and the pellet was transferred into a new tube with $75 \%$ ethanol $(1 \mathrm{~mL})$. Then, the tube was vortexed gently and centrifuged at $7500 \times \mathrm{g}$ for 5 min at $4{ }^{\circ} \mathrm{C}$. The RNA pellet was air-dried, suspended in RNase-free water and water bathed at $60^{\circ} \mathrm{C}$ for $10 \mathrm{~min}$. An Agilent 2100 Bioanalyzer was used to evaluate the quantity and quality of the total RNA of each sample.

\section{Transcriptome library construction and sequencing}

We used both paired-end and single-end strategies for transcriptome sequencing. The cDNA library of each sample was constructed using the TruSeq RNA Sample Preparation Kit v2 (Illumina) and sequenced on the Illumina HiSeq 2000 platform, according to protocols. Briefly, equal amount $(20 \mu \mathrm{g})$ of total RNA (RIN > 8.0) of each sample was used to enrich the mRNAs using the Dynal Oligo(dT) beads (Invitrogen). The mRNAs were then chemically fragmented into $\sim 200 \mathrm{nt}$ fragments using divalent cations (Elute/Prime/Fragment Mix buffer, Illumina) under elevated temperature, followed by the cDNA synthesis. The cDNA fragments were end-repaired using End Repair Mix (Illumina) and purified. Then, we used TruSeq Paired-End Cluster Kit v3 (Illumina PE-401-3001) and TruSeq SBS HS Kit v3 (Illumina FC-401-3001) to generate the final cDNA libraries for paired-end $(90 \mathrm{bp} \times 2)$ and single-end (50 bp) sequencing, respectively, according to the protocols [22]. Mixture of CK samples was named as CK-MIX and mixture of DTS samples was named as DTS-MIX. DTS-MIX and CK-MIX were processed with paired-sequencing while DTS-R1, DTS-R2, DTS-R3, CK-R1, CK-R2 and CK-R3 were processed with single-end sequencing.

\section{De novo analysis and transcriptome annotation}

Raw reads from the paired-end sequencing were cleaned by removing low quality reads and reads with adaptors or ambiguous base ' $\mathrm{N}$ '. The resulted high-quality reads were then quality controlled using FASTQC ((http:// www.bioinformatics.babraham.ac.uk/projects/fastqc/).

Trinity software (v2.4.0) was used for the de novo transcriptome analysis with default parameters [23]. Then, using the superTranscripts function provided by Trinity we constructed the possible 'gene' sequences of sugarcane [24].

To perform the transcriptome annotation, we first identified likely coding sequences in the sugarcane transcriptome using TransDecoder (https://transdecoder.github.io/) under default parameters. Then, Trinotate (v2.0.2, available at http://trinotate.github.io/) was used to annotate the deduced proteins. Briefly, the sugarcane deduced proteins were searched against the UniProtKB/Swiss-Prot database to identify known protein sequences. HMMER was used to predict functional domains of the deduced proteins by mapping them to the PFAM database [25]. Next, SignalP [26], RNAMMER [27] and TMHMM Sever 2.0 [28] were used to annotate potential signal peptides, ribosomal RNA transcripts and transmembrane domains, respectively, for the assembled transcriptome. The deduced proteins were also searched against the EggNOG database (v 4.1, http:// eggnogdb.embl.de/) to identify known proteins in EuKaryotic Orthologous Groups (KOG), Clusters of Orthologous Groups (COGs), and non-supervised orthologous groups (NOGs) [29]. All the above annotations were loaded to a Trinotate SQLite database and a final annotation was produced. The cut-off of e-values for best hits was set to $1 \mathrm{e}-5$.

\section{Gene ontology and KEGG pathway annotation}

We also annotated the assembled genes related to Gene Ontology (GO) and Kyoto Encyclopedia of Genes and Genomes (KEGG) pathway. In brief, all the assembled genes were search against NCBI non-redundant (NR), UniProt and KEGG pathway databases using the BLAST software [30]. Cut-offs and other filters were applied to select the best hits, as previously described [12].

\section{Reads alignment and gene expression profiling}

The Trinity transcripts were quantified using the RSEM (RNA-Seq by Expectation-Maximization) method [31]. After data cleaning described above, both paired-end and single-end sequencing reads were aligned to the assembled transcriptome using Bowtie2 [32]. Then, RSEM tool was used to identify the gene expression levels in all samples [31]. In this study, TPM (transcripts per million reads) method was used for normalization and lowly expressed genes $(<5$ TPM) were filtered. Recommended parameters by Trinity were used for Bowtie 2 and RSEM. 


\section{Differential expression analysis}

Differentially expressed genes in DTS samples relative to CK were identified using the edgeR software [33]. Following parameters were used to select the differentially expressed genes: i) $>5$ TPM in at least one sample, ii) $\log 2 \mathrm{FC}$ ( $\log 2$ fold change) $>1$ (up-regulated) or $\log 2 \mathrm{FC}$ $<-1$ (down-regulated), iii) $p$-value $<0.5$ and iv) FDR (false discovery rate) $<0.05$.

\section{Functional analysis}

Enrichment of GO terms and KEGG pathways was analyzed to predict the functions of candidate genes. Fisher's exact test was used to calculate the $p$-value which represents the significance of enrichment, and an $\mathrm{R}$ package named 'q-value' was used to correct the $p$-value for each GO term/ KEGG pathway and control the false discovery rate. Significant GO terms and KEGG pathways were selected if the $p$-value $<0.05$ and $q$-value $<0.05$. GO terms and KEGG pathways not related to plant bio activities were filtered.

\section{qRT-PCR}

Quantitative real-time PCR (qRT-PCR) experiment was performed to confirm the expression patterns in the leaves, following the protocol [12]. In brief, the total RNA was extracted using TRIzol reagent (Invitrogen) and quality-controlled using the Agilent 2100 Bioanalyzer. Primer3 (http://bioinfo.ut.ee/primer3-0.4.0/) was used to predict the forward and reverse primers for 9 randomly selected genes and the endogenous control (actin). All the primers were synthesized at BGI-Shenzhen. The cDNA synthesis and qRT-PCR experiments were performed, as previously described [12]. Three reactions were conducted for each candidate gene in every sample. Then, the average $\mathrm{Ct}$ (cycle threshold) was calculated and $\Delta \mathrm{Ct}$ was used to show the expression level of each candidate gene (relative to actin). $\Delta \Delta \mathrm{Ct}$ method was used to show the different expression of a gene in drought-stressed sugarcane leaves, compared to CK, as described [12].

\section{miRNA target prediction}

miRNA target prediction was performed using the plant miRNA target prediction software psRobot with default parameters [34].

\section{Results}

\section{De novo analysis of Saccharum narenga leaf transcriptome}

Six Saccharum narenga plants were obtained from a barren mountain in Guangxi, China and transplanted into a greenhouse of Guangxi Academy of Agricultural Sciences. During the maturity period, three of them (DTS) were not watered for 22 days and the rest (CK) were treated with sufficient water. One leaf of each plant was randomly selected for total RNA isolation and subsequent transcriptome sequencing. Two paired-end $(2 \times$ $90 \mathrm{bp}$ ) libraries (CK-MIX and DTS-MIX) and six single-end (50 bp) libraries for CK (CK-R1, CD-R2 and CK-R3) and DTS (DTS-R1, DTS-R2 and DTS-R3) samples were sequenced on the Illumina HiSeq 2000 platform. The paired-end sequencing produced a total of $15.4 \mathrm{G}$ data ( $\sim 171$ million raw reads). After data cleaning, we obtained 156.8 million clean reads and processed the de novo analysis using Trinity software (v2.4.0) [23] (Table 1). The assembly produced a total of 312,800 transcripts, which contain $361,982,856$ bases (534 $\mathrm{M}$ in size) (Table 1). The N50 statistic was 1771 , which meant that more than $50 \%$ of the transcripts were longer than 1771 bp. Then, identification of the superTranscripts function found that these transcripts were from 104,644 genes that contain 89,330,093 bases (122 $\mathrm{M}$ in size) (Table 1). The N50 statistic was 1605 while the average length of all the genes was $853.66 \mathrm{bp}$ (Table 1). The length distribution of all the assembled sugarcane genes was shown in Fig. 1, which indicated that $16.8 \%$ of the total transcripts and $18.6 \%$ of the total genes were longer than $2000 \mathrm{bp}$. This is the first time to study the Saccharum narenga leaf transcriptome, so it is difficult to evaluate the number of transcripts/genes in Saccharum narenga due to the missing information of its genome sequence and annotation.

\section{Annotation of the assembled sugarcane genes}

We next annotated the assembled sugarcane genes by mapping them to multiple databases, such as NCBI non-redundant (NR), UniProt, GO and KEGG databases. Fig. 2a shows that 29,391 (28.09\%), 30,814 (29.45\%), 28,397 (27.14\%) and 21,789 (20.82\%) gene sequences were aligned to the UniProt, NR, GO and KEGG databases, respectively. In addition, 3 rRNA sequences were predicted by RNAMMER [27]. Further, in the NR mapping results we retrieved and counted the genes aligned to different species and the top 10 species aligned by the assembled sugarcane genes were listed in Fig. 2b, which

Table 1 Overview of the assembled sugarcane transcriptome

\begin{tabular}{lcc}
\hline Type & CK_MIX & DTS_MIX \\
\hline Total paired-end reads & $79,172,916$ & $77,639,556$ \\
Total trinity transcripts & 312,800 \\
N50 (transcripts) & 1771 \\
Total assembled bases (transcripts) & $361,982,856$ \\
Total trinity genes & 104,644 \\
N50 (genes) & 1605 \\
Average length (genes) & 853.66 \\
Total assembled bases (genes) & $89,330,093$ \\
GC (\%) & 47.36 \\
\hline
\end{tabular}




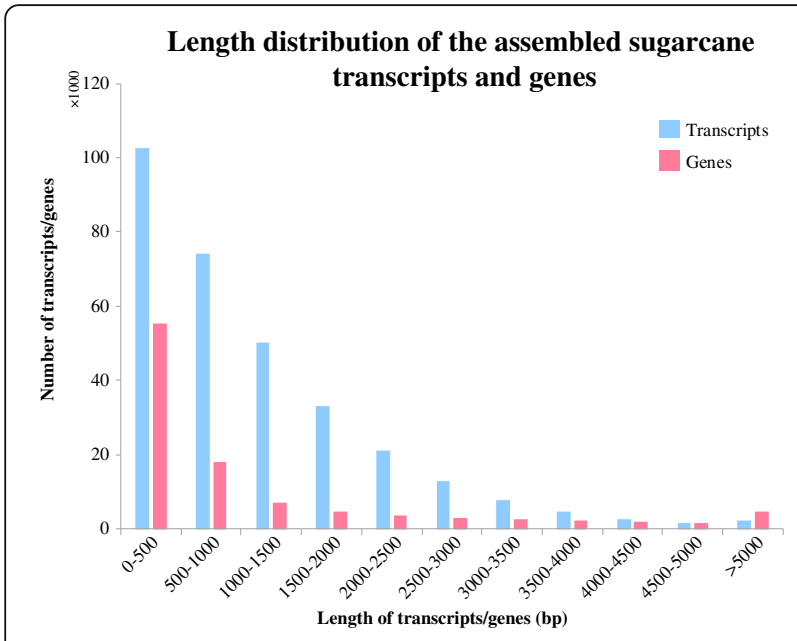

Fig. 1 Length distribution of the assembled sugarcane transcripts and genes

showed that 22,392 genes, $21.40 \%$ of the assembled sugarcane genes, were aligned to Sorghum bicolor, followed by Zea mays (8997 genes), Setaria italica (3414 genes), and Oryza sativa Japonica Group (1694 genes). GO annotation (Additional file 1) revealed that 13,157, 13,920 and 10,363 genes were involved in "cellular process", "metabolic process" and "catalytic activity", respectively. In addition, "developmental process", "growth" and "response to stimulus" were identified to involve 1553, 278 and 3438 genes, respectively. Top five KEGG pathways involved by the sugarcane genes were "metabolic pathways" (ko01100, 4961 genes), "biosynthesis of secondary metabolites" (ko01110, 3267 genes), "plant-pathogen interaction" (ko04626, 1786 genes), "neurotrophin signaling pathway" (ko04722, 1581 genes) and "apoptosis" (ko04210, 1486 genes).

Further, TransDecoder (https://transdecoder.github.io/ ) was used to identify 192,553 likely proteins encoded by 35,424 of the assembled sugarcane genes (Fig. 2a). It was found that 113,817 (58.59\%), 107,455 (55.81\%), 140,761 (73.10\%) and 110,153 (57.21\%) of the deduced proteins were aligned to UniProt, GO, KEGG and Pfam databases, respectively. SignalP [26] identified 3 proteins containing signal peptides and TMHMM [28] identified 30,252 proteins that were highly similar to membrane proteins (Fig. 2a). Then, the deduced proteins were aligned to EggNOG database (v4.1, http://eggnogdb.embl.de). In total, 55,323 deduced proteins were annotated into 1374 COGs, 41 KOGs and 333 NOGs. We found that the most significant known COG category was "signal transduction mechanisms", which involved 11,748 deduced proteins, followed by "post-translational modification, protein turnover, and chaperones" that involved 5782 deduced proteins (Fig. 2c). Meanwhile, 12,304 deduced proteins (Fig. 2c) were annotated as "function unknown". Different annotation perspectives of the assembled sugarcane transcriptome will help understand of the process of sugarcane leaves in response to water deficit. In addition, the reasons of some genes without encoding capacity require to be explored with more experiments [12]. In total, 39,716 of the assembled genes were annotated to be similar to other species while 22,509 of them were predicted to encode proteins. This means that 12,915 of the assembled genes were predicted to encode proteins that have not been reported. More experiments are required to study the novel genes/proteins and their functions in Saccharum narenga under water stress.

\section{Gene expression profiling}

To profile the gene expression in CK and DTS samples, both paired-end and single-end sequencing reads were aligned to the assembled sugarcane gene sequences using Bowtie2 [32]. Then, RSEM [31] was used to estimate the abundance of each gene in the samples and TPM (transcripts per million reads) method was used for normalization. After lowly expressed genes $(<5$ TPM) were filtered, a total of 30,297 genes were identified across all samples (Additional file 2). In detail, 24,598 and 25,584 genes were identified in CK (CK-R1: 22,151, CK-R2: 18,982, CK-R3: 21,126) and DTS (DTS-R1: 22,565; DTS -R2: 22,485; DTS -R3: 22,623) samples, respectively, using single-end sequencing (Fig. 2d). And 22,068 and 23,797 genes were identified in CK-MIX and DTS-MIX, respectively, by paired-end sequencing (Fig. 2d). A heat map (Fig. 2e) showed that the correlation between replicates was high and DTS samples were distinct from CK samples based on both paired-end and single-end sequencing data.

\section{Differential expression analysis}

We next used edegR [33] to identify differentially expressed genes (DEGs) in sugarcane leaves in response to water deficit. As shown in Fig. 3a, single-end and paired-end sequencing identified 4551 (2327 up-regulated and 2224 down-regulated) and 4212 (2268 up-regulated and 1944 down-regulated) genes, respectively, differentially expressed in DTS samples, compared to CK (Additional file 3). It was found that the expression patterns of 3389 (1772 up-regulated and 1617 down-regulated) genes were consistent in paired-end and single-end sequencing (Fig. 3a, Additional file 4). We also used a heat map to show the expression changes of genes in sugarcane leaves in response to water deficit (Fig. 3b). Among the DTS up-regulated genes 843 were lowly expressed $(<5$ TPM) in CK. We showed the highly expressed (>100 TPM) DTS-specific genes in Fig. 3c, such as TRINITY_DN24753_c0_g2 encoding transposon Tf2-9 polyprotein, TRINITY_DN21466_c1_g1 encoding non-specific 

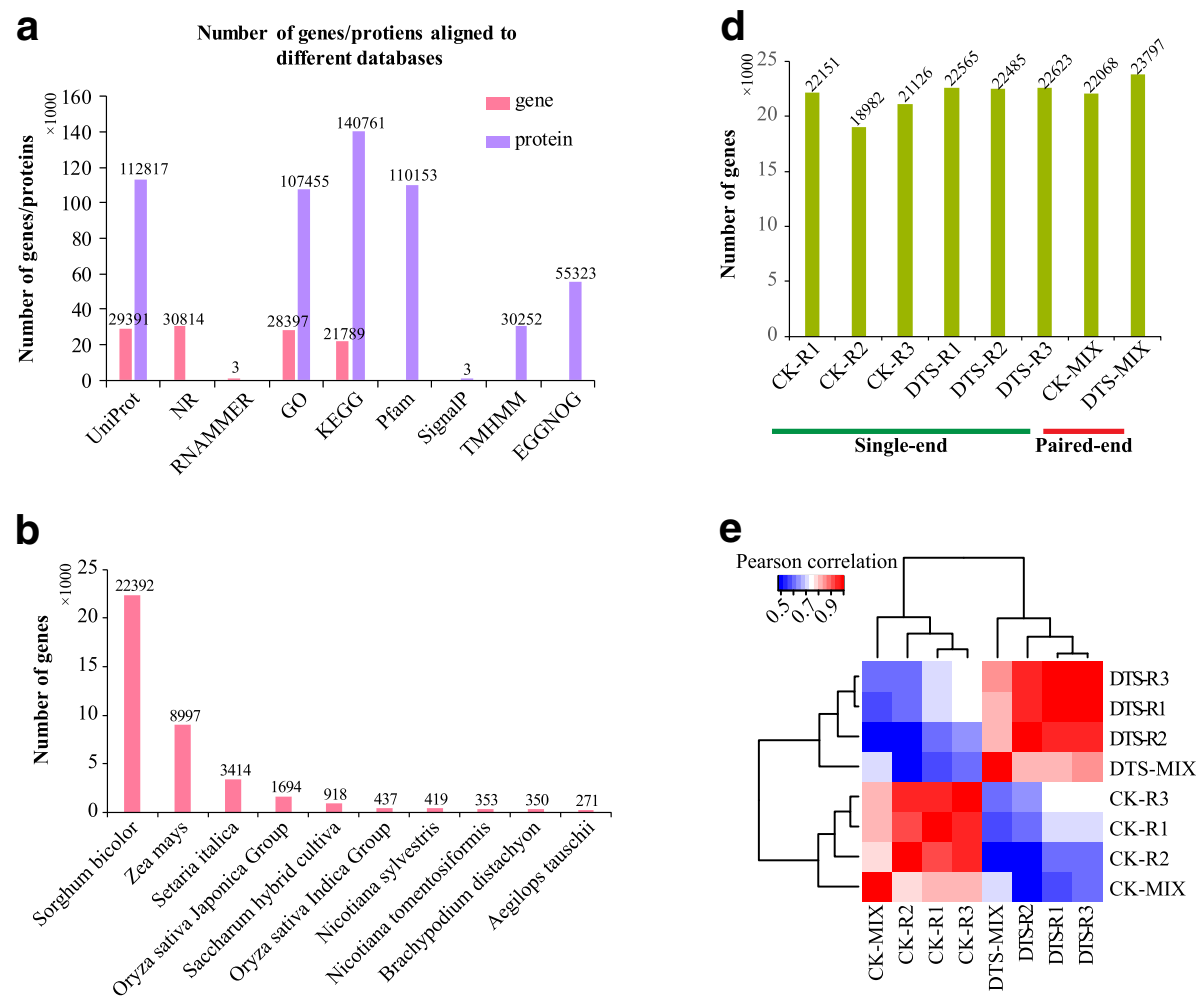

C

COG annotation categories

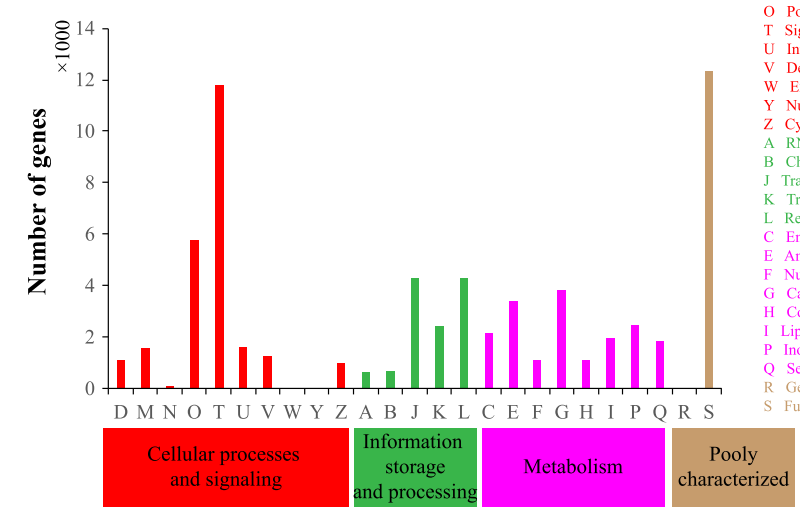

D Cell cycle control, cell division, chromosome partitioning Cell wall/membrane/envelope biogenesis Cell motility

-translational modification, protein turnover, and chaperones ignal transduction mechanisms

Dacellular trafficking, secretion, and vesicular transport

Extracellular structure

Nuclear structur

Cytoskeleton

RNA processing and modification

structure and dynamics

thosomal structure and biogenesis

Transcription

(n)

nsport and metabolis

transport and metabolism

Corbhydate transpor and melabolis

Coenzyme transport and metabolis

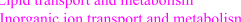

Gcondary metabolites biosynthesis,

General function prediction only

characterized

Fig. 2 Annotation of the assembled sugarcane transcriptome and gene expression profiling. a Number of genes and predicted proteins aligned to different databases. $\mathbf{b}$ Distribution of species aligned by the assembled sugarcane genes. c COG annotation for the assembled sugarcane genes. $\mathbf{d}$ Number of genes identified in each sample (> 5 TPM). e Heat map of sample correlation based on the gene expression profile

lipid-transfer protein 2, TRINITY_DN19178_c0_g1 encoding low molecular mass early light-inducible protein HV60, TRINITY_DN19705_c1_g1 encoding dehydrin DHN1, TRINITY_DN18684_c0_g1 encoding late embryogenesis abundant (LEA) protein and TRINITY_DN14391 _c0_g1 encoding low temperature-induced protein lt101.2. In Fig. 3d, we showed top 10 genes (expression ranged from 28.48 TPM to 108.75 TPM) that were identified in CK samples but lowly expressed ( $<5$ TPM) in DTS samples. Except for the genes only identified in DTS or
CK samples, we also identified the differentially expressed genes that encode various protein products, including $\mathrm{ABC}$ transporter, auxin response factor, light-regulated protein, chlorophyll a-b binding protein and galactinol-sucrose galactosyltransferase.

\section{Functional analysis}

To gain insights of sugarcane leaf genes in response to water deficit, functional analysis was performed to identify enriched GO terms and KEGG pathways involved by 

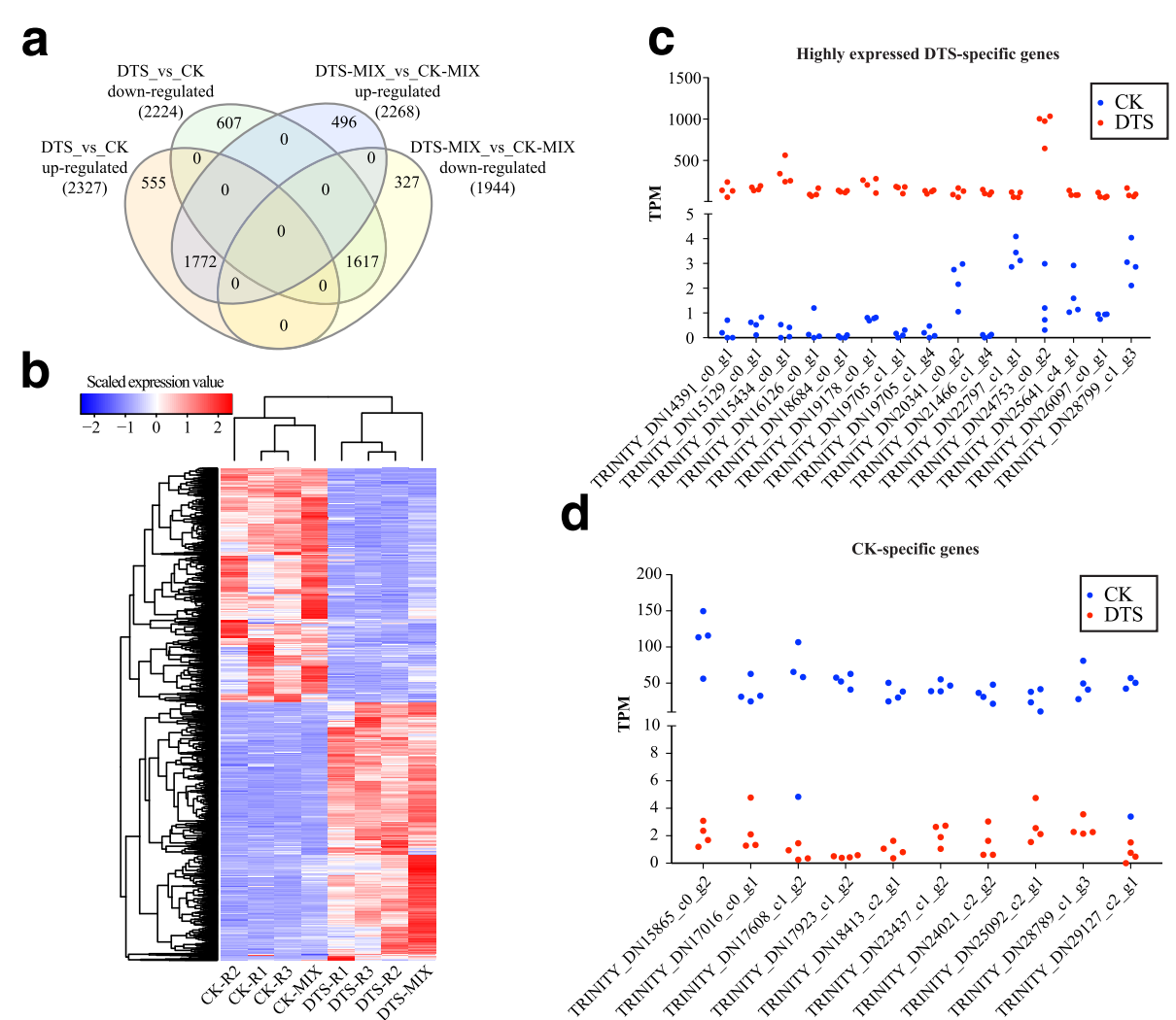

Fig. 3 Differential expression analysis. a Venn diagram of DEGs identified by paired-end and single-end sequencing technologies. b Heat map of the DEG expression levels. $\mathbf{c}$ Highly expressed genes (> 100 TPM) exclusively identified in DTS samples. $\mathbf{d}$ Top 10 highly expressed genes identified exclusively in CK samples

the DEGs. Among the significant GO terms (Table 2), we found that 19 genes were involved in "response to blue light" (GO:0009637), 12 genes were related to "chloroplast part" (GO:0044434) and 9 genes were enriched in "negative regulation of defense response" (GO:0031348). Next, we used a bubble plot to show the significant KEGG pathways involved by the DGEs (Fig. 4). It showed 537 DEGs were involved in "metabolic pathways" (ko01100), which is the most significant. Interestingly, we identified 40 DEGs involved in "circadian rhythm - plant" (ko04712) that is related to environmental adaptation. In addition, 161 and 107 DEGs were involved in the pathways of "apoptosis" (ko04210) and "plant hormone signal transduction" (ko04075), respectively. In addition, plant hormone related pathways "flavonoid biosynthesis" (ko00941), "steroid hormone biosynthesis" (ko00140), "zeatin biosynthesis" (ko00908) and "sesquiterpenoid and triterpenoid biosynthesis" (ko00909) were identified to involve 28, 17, 12 and 16 DEGs, respectively. Although it is not clear about the functions of these pathways in sugarcane leaves in response to water deficit, previous studies have shown that metabolism, auxin, abscisic acid (ABA), cytokinins and ethylene are related with cell growth and cell death in plants [35-37].

\section{Sugarcane genes in response to water deficit}

We next analyzed the DEGs in different categories that might be related to sugarcane leaves in response to water deficit, such as aquaporin, LEA, auxin related proteins, transcription factors, heat shock proteins, chlorophyll a-b binding proteins and some other proteins. In Table 3, we showed the statistics of these gene families in this study, including the number of genes identified and the number of dysregulated in DTS samples compared to CK.

\section{Aquaporin}

In this study, we identified 60 genes encoding aquaporin (Table 3 ), of which 11 were differentially expressed (8 up-regulated and 3 down-regulated) in DTS samples relative to CK (Additional file 4). Interestingly, the regulation of genes varied among different aquaporin subfamilies (TIP, NIP and PIP). In general, genes encoding NIPs and PIPs were up-regulated in sugarcane leaves in response to the water-deficit stress while genes encoding TIPs were down-regulated (Fig. 5a).

\section{LEA}

There were 13 genes encoding LEA in the assembled sugarcane leaf transcriptome (Table 3). We identified 6 
Table 2 GO enrichment analysis of DEGs in DTS samples compared to CK

\begin{tabular}{|c|c|c|c|c|c|}
\hline Type & ID & Term & Number & Enrich_factor & $p$-value \\
\hline \multirow[t]{17}{*}{ Biological process } & GO:0009886 & post-embryonic morphogenesis & 1 & 1.09 & 5.19E-06 \\
\hline & GO:0015977 & carbon fixation & 4 & 3.91 & $2.57 \mathrm{E}-05$ \\
\hline & GO:0015786 & UDP-glucose transport & 2 & 0.75 & $5.55 \mathrm{E}-05$ \\
\hline & GO:0009695 & jasmonic acid biosynthetic process & 5 & 0.50 & 9.06E-05 \\
\hline & GO:0031348 & negative regulation of defense response & 9 & 0.62 & 0.0001101 \\
\hline & GO:0006014 & D-ribose metabolic process & 3 & 1.38 & 0.0001256 \\
\hline & GO:1902582 & single-organism intracellular transport & 1 & 0.72 & 0.0002646 \\
\hline & GO:0045491 & xylan metabolic process & 2 & 1.47 & 0.0003353 \\
\hline & GO:0009637 & response to blue light & 19 & 1.82 & 0.0003557 \\
\hline & GO:0086010 & membrane depolarization during action potential & 2 & 2.79 & 0.0005124 \\
\hline & GO:0048869 & cellular developmental process & 4 & 6.18 & 0.0005507 \\
\hline & GO:0044723 & single-organism carbohydrate metabolic process & 5 & 2.07 & 0.0005799 \\
\hline & GO:0010236 & plastoquinone biosynthetic process & 2 & 1.89 & 0.000751 \\
\hline & GO:0009750 & response to fructose & 5 & 0.45 & 0.000751 \\
\hline & GO:0046483 & heterocycle metabolic process & 1 & 1.13 & 0.0007543 \\
\hline & GO:0051726 & regulation of cell cycle & 2 & 0.28 & 0.0008238 \\
\hline & GO:0055088 & lipid homeostasis & 2 & 0.69 & 0.0009208 \\
\hline Molecular function & GO:0015254 & glycerol channel activity & 9 & 1.89 & 0.0003135 \\
\hline \multirow[t]{2}{*}{ Cellular component } & GO:0009522 & photosystem I & 19 & 2.10 & 0.001842 \\
\hline & GO:0044434 & chloroplast part & 12 & 2.35 & 0.004865 \\
\hline
\end{tabular}

LEA genes $>5$ TPM in all samples and all of them were up-regulated in the sugarcane leaves in response to water deficit. Up-regulated LEA genes were annotated to have the capacity to encode proteins LEA5D_GOSHI, LEA3_MAIZE, LEA14_GOSHI, LEA5_CITSI and LEA34_GOSHI. It is notable that 4 out of the 6 LEA DEGs were not detected (<5 TPM) in CK (Fig. 5b).

\section{Auxin-related protein}

We identified 175 genes encoding auxin-related proteins (Additional file 4), including auxin response factors (ARFs), auxin-responsive proteins (IAAs), auxin efflux carrier components, auxin-repressed proteins, auxin-binding proteins and auxin-induced proteins. However, only 15 of them were differentially expressed in DTS samples compared to CK. They include 2 genes encoding ARFs, 2 genes encoding auxin-induced proteins and 11 genes encoding IAAs. It is interesting that DEGs encoding ARFs and auxin-induced proteins (TRINITY_DN26758_c0_g1 and TRINITY_DN18434_c1_g4) were up-regulated in sugarcane leaves in response to water deficit, however, genes encoding IAAs were down-regulated, generally, in DTS samples compared to CK (Fig. 5c).

\section{Transcription factor}

There were 882 sugarcane genes that have the capacity of encoding TFs, of which 106 were differentially expressed
(Table 3). These 106 TF DEGs include 70 up-regulated genes, which can encode bZIP TF TRAB1, ERFs (ethylene-responsive TFs), heat stress TFs, NAC TFs, WRKY TFs and DIVARICATA TFs, and 36 down-regulated genes, which can encode GATA TFs, Nuclear TF Y subunits, bHLH TFs, WRKY TFs and TCP TFs. A heat map of their expression values in DTS and CK samples were shown in Fig. 5c. ERFs and heat stress TFs were identified to be induced by water deficit in sugarcane leaves while TCP TFs were suppressed. It is difficult to determine the regulation of WRKY TFs in sugarcane leaves in response to water deficit. More experiments are required to explore the functions of WRKY TFs, as well as other TF genes.

\section{Heat shock protein}

We next analyzed the expression changes of heat shock proteins (HSPs) in sugarcane leaves in response to water deficit. Among the 157 HSP genes, 17 (10.8\%) were differentially expressed (16 up-regulated and 1 down-regulated) in DTS samples compared to CK (Table 3, Additional file 4). Compared to the up-regulated HSP genes, the down-regulated HSP gene TRINITY_DN16108_c0_g1 was lowly expressed in both DTS (ranged from 5.71 to 11.24 TPM) and CK (ranged from 2.29 to 3.65 TPM) samples (Fig. 5e). Interestingly, HSP $70 \mathrm{kDa}$ proteins are the largest family encoded by the differentially expressed HSP genes. 


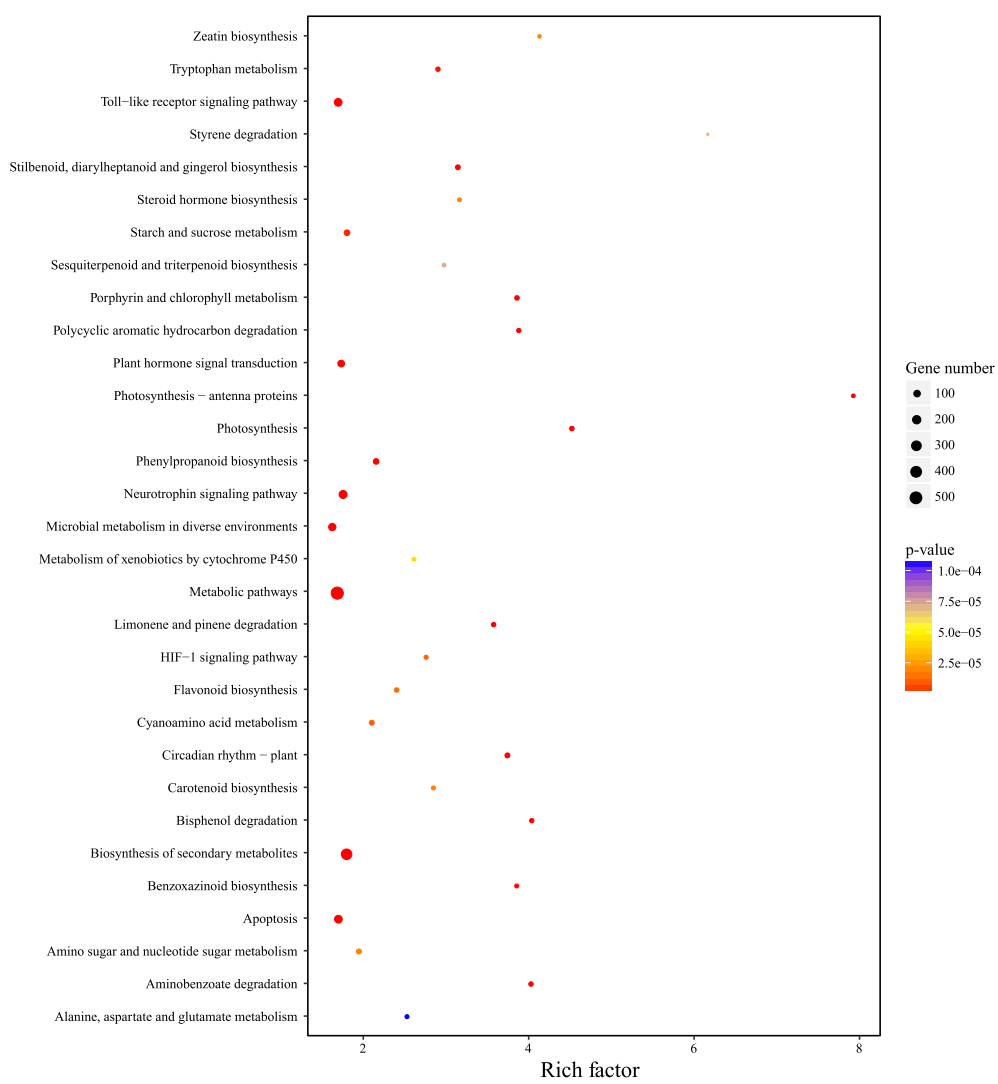

Fig. 4 KEGG enrichment analysis of the differentially expressed genes

Table 3 Important gene families in response to water deficit in sugarcane leaves

\begin{tabular}{lllll}
\hline Family & annotated & $\begin{array}{l}\text { identified } \\
(>5 \text { TPM })\end{array}$ & $\begin{array}{l}\text { up- } \\
\text { regulated }\end{array}$ & $\begin{array}{l}\text { down- } \\
\text { regulated }\end{array}$ \\
\hline Aquaporin & 60 & 22 & 8 & 3 \\
LEA & 13 & 6 & 6 & 0 \\
auxin-related & 175 & 72 & 7 & 7 \\
heat shock protein & 68 & 33 & 5 & 1 \\
transcription factor & 882 & 425 & 70 & 36 \\
$\begin{array}{l}\text { chlorophyll a-b } \\
\text { binding protein }\end{array}$ & 59 & 27 & 0 & 22 \\
$\begin{array}{l}\text { light-regulated } \\
\text { proteins }\end{array}$ & 1 & 1 & 0 & 1 \\
$\begin{array}{l}\text { non-specific } \\
\text { lipid-transfer protein } \\
\text { disease resistance }\end{array}$ & 40 & 14 & 5 & 2 \\
protein & 1458 & 456 & 14 & 45 \\
$\begin{array}{l}\text { ribosomal protein } \\
\text { dehyrin }\end{array}$ & 809 & 333 & 2 & 18 \\
\hline
\end{tabular}

\section{Light-harvesting chlorophyll $a-b$ binding protein}

Genes encoding light-harvesting chlorophyll $a-b$ binding proteins (LHCB) are the highest expressed gene family in the sugarcane leave transcriptome (Additional file 2). In total, 59 LHCB genes were annotated in the assembled sugarcane transcriptome and $22(37.29 \%)$ of them were down-regulated in DTS samples compared to CK (Table 3, Fig. 5f). Three LHCB genes were very abundant $(>10,000$ TPM) in CK samples, such as TRINITY_DN20351_c2_g1 (CB23_ORYSJ), TRINITY_DN25403_c0_g1 (CB23_ORYSJ), TRINITY_DN27533_c1_g1 (CB2G_SOLLC). Because of the water-deficit stress, they were dropped by $\sim 4$ times to $<4500$ TPM (Additional file 4).

\section{Other water-deficit associated genes}

We also observed the expression patterns of some other gene families that might relate to the drought tolerance of sugarcane leaves, such as genes encoding light-regulated protein, non-specific lipid-transfer protein, putative disease resistance proteins, dehydrin and ribosomal proteins (Fig. 5g, Additional file 4). The disease resistant protein family might be the largest gene family identified in this study. We identified 1458 genes that can encode disease resistance proteins and 456 were expressed more than 5 TPM. Of them, 14 up-regulated and 45 down-regulated 


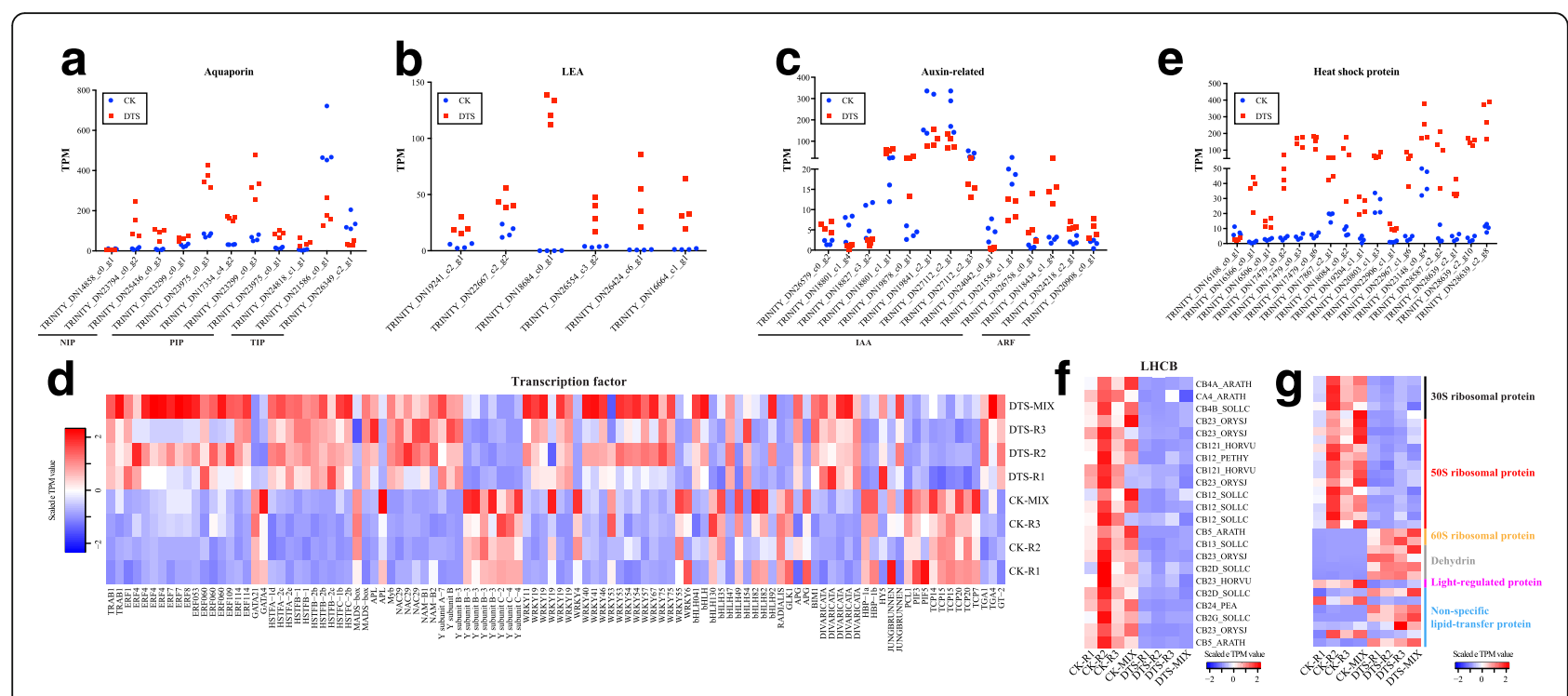

Fig. 5 Sugarcane genes in response to the water-deficit stress. We investigated the expression levels of DEGs in several families, such as a aquaporin, $\mathbf{b}$ LEA, $\mathbf{c}$ auxin-related protein, $\mathbf{d}$ transcription factor, e heat shock protein, $\mathbf{f}$ light harvest chlorophyll a-b binding protein, and $\mathbf{g}$ some other protein families

genes were identified in DTS samples compared to CK (Table 3, Additional file 4). It is interesting that genes encoding $30 \mathrm{~S}$ and $50 \mathrm{~S}$ ribosomal proteins were up-regulated while genes encoding 60S ribosomal proteins were down-regulated. This indicates they may have diverse functions in sugarcane leaves in response to water deficit. All the 4 genes encoding dehydrin proteins were up-regulated while the only DEG (TRINITY_DN28547_c1_g1) encoding light-regulated protein was down-regulated in DTS samples compared to CK (Additional file 4). Among the DEGs encoding non-specific lipid-transfer proteins, 2 were down-regulated while 5 were up-regulated in DTS samples relative to CK (Additional file 4).

\section{qRT-PCR}

We next used qRT-PCR to validate the gene expression levels in sugarcane leaves in response to water deficit. Forward and reverse primers for 9 randomly selected genes and internal control (actin) were designed using Primer3 (http://bioinfo.ut.ee/primer3-0.4.0/) and can be accessed in Additional file 5. For each candidate gene, three reactions were performed in each sample $(n=3 \times 3)$ and $\Delta \Delta \mathrm{Ct}$ method was used to present the expression change of a gene in DTS samples compared to CK. As shown in Fig. 6, the expression patterns of all these nine genes identified by qRT-PCR were consistent to those identified by both single-end and paired-end RNA sequencing. Except for TRINITY_DN23141_c2_g1, the other eight genes were up-regulated significantly $(\log 2 \mathrm{FC}>1)$.

\section{miRNA regulation of DEGs}

To understand the regulation of DEGs by miRNAs, we obtained a total of 412 (261 conserved and 151 novel
miRNA) sugarcane miRNAs sequences from a recently published study [38]. Then, psRobot was used to predict the target genes of sugarcane miRNAs in the sugarcane genes assembled in this study. We identified a total of 6577 sugarcane leaf genes that could be regulated by the sugarcane miRNAs. Further, 553 genes were found with differential expression between DTS and CK samples (Additional file 6). These 553 DEGs can be regulated by 175 sugarcane miRNAs, including 89 conserved and 86 novel sugarcane miRNAs. In Table 4, several conserved miRNAs and their target genes were shown, which were dysregulated in sugarcane leaves in response to water deficit. Among them, miR164 that regulates mRNAs encoding NAC domain-containing proteins has been previously reported in sugarcane in response to drought

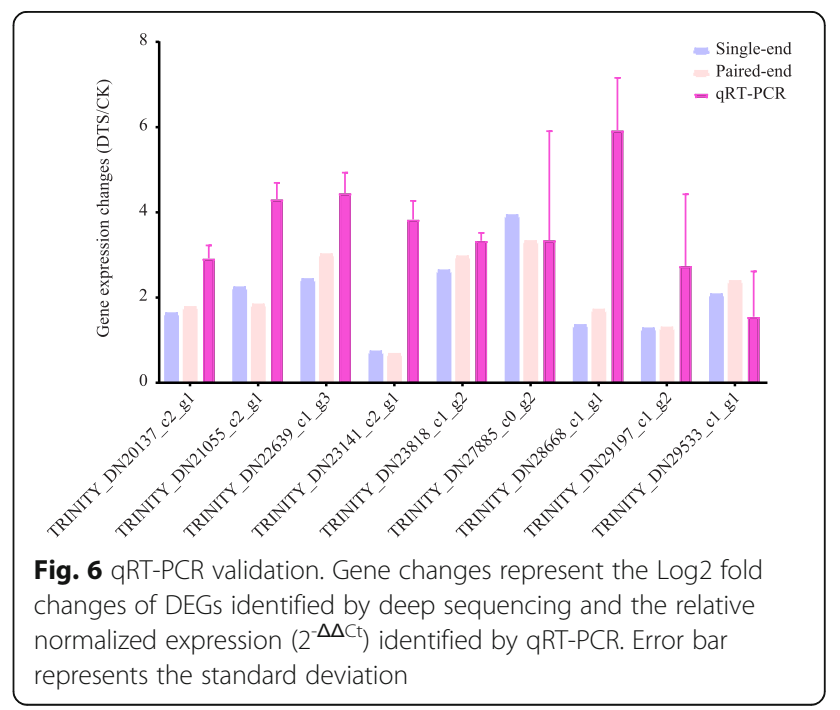




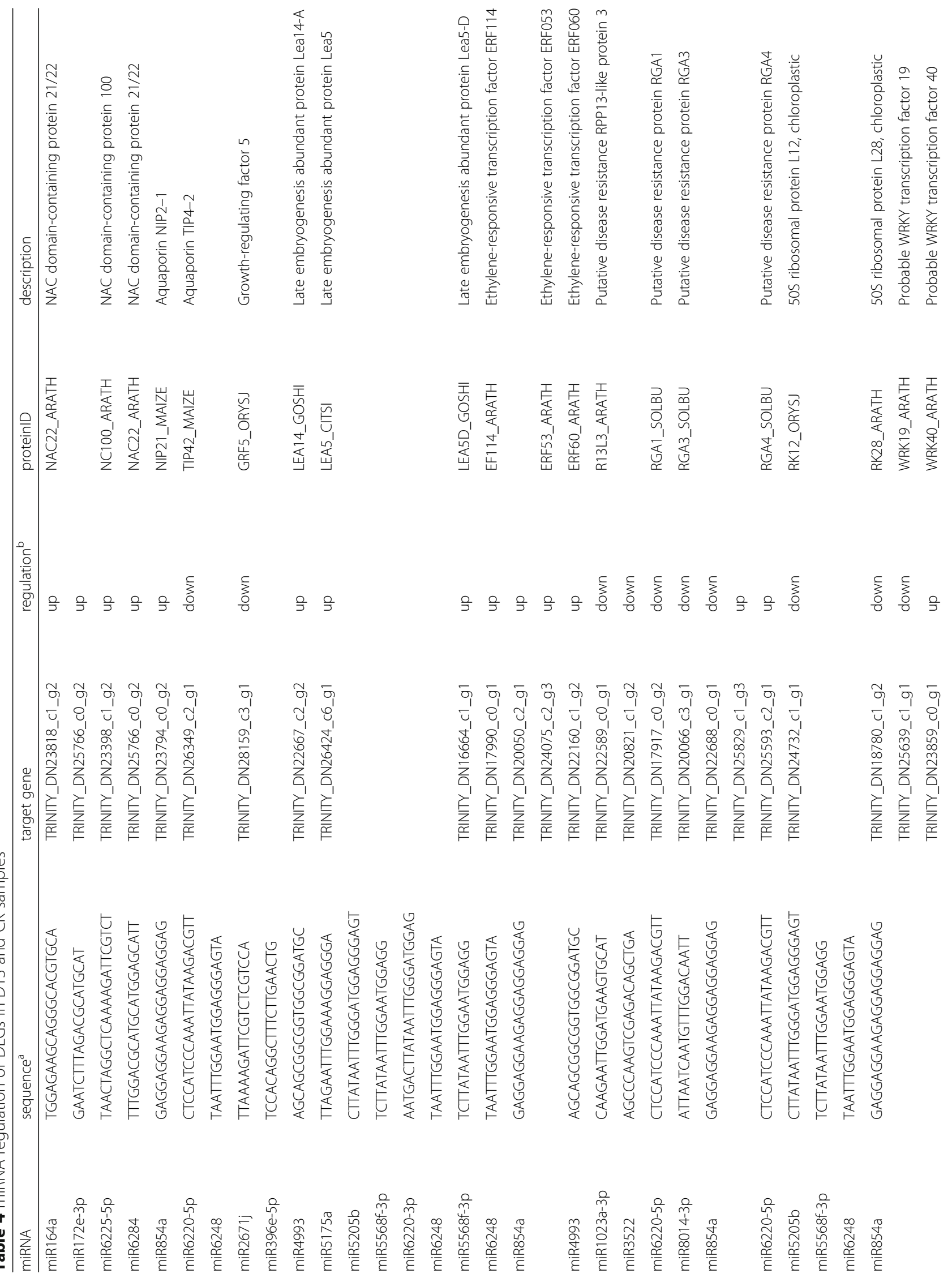




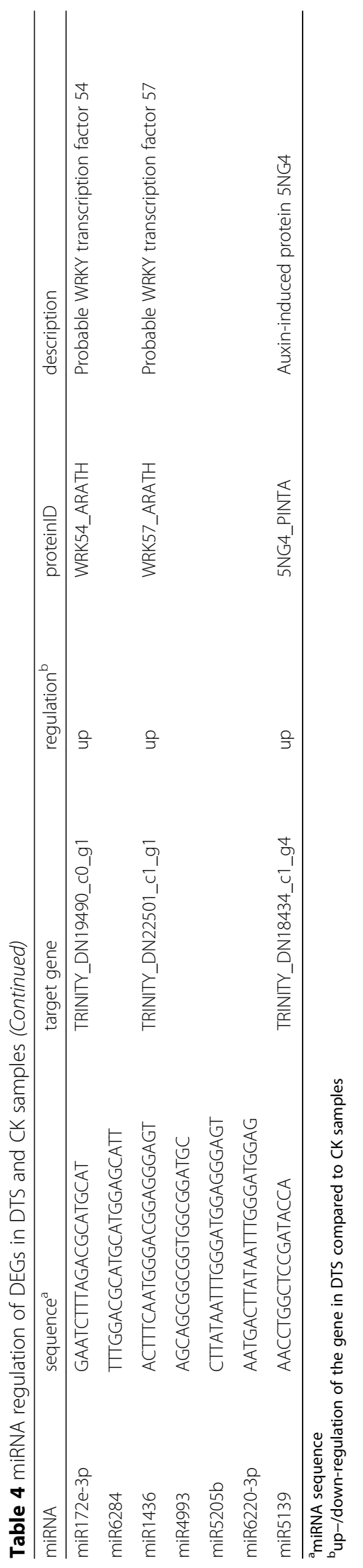


stress [39]. In addition, we identified another three miRNAs (miR172e-3p, miR6225-5p and miR6284) that can regulate the expression of NAC domain-containing protein genes. It is interesting that 7 miRNAs (miR1023a-3p, miR3522, miR6220-5p, miR8014-3p, miR854a, miR854a and miR6220-5p) were identified to target genes encoding disease resistant proteins, which were down-regulated in sugarcane leaves in response to water deficit. It is notable that 8 miRNAs were predicted to regulate WRKY TF genes. Further experiments are required to explore the miRNA regulation in sugarcane leaves and their target gene expression, especially miR6284 and miR854a (Table 4), because they can interact with multiple gene families, such as NAC domain-containing protein, LEA, ethylene-responsive TF, disease resistance protein, ribosomal protein and WRKY TF.

\section{Discussion}

In this study, we used paired-end and single-end sequencing technologies to investigate the gene expression profiles of sugarcane leaves in response to water deficit. This is the first transcriptome study of Saccharum narenga species using the deep sequencing technology. We identified 104,644 sugarcane leaf genes ( N50 $=1605 \mathrm{bp})$, of which $21.40 \%$ can be aligned to Sorghum bicolor. In DTS and CK samples we identified 30,297 genes ( $>5$ TPM), of which single-end sequencing identified 4551 (2327 up-regulated and 2224 down-regulated) DEGs while paired-end sequencing identified 4212 (2268 up-regulated and 1944 down-regulated) DEGs in DTS samples, compared to CK. Functional analysis showed that DEGs in DTS and CK were involved in "circadian rhythm - plant" and "plant hormone signal transduction". Our further analysis of gene expression patterns revealed that several gene families, such as aquaporin, LEA, auxin related protein, TF, heat shock protein and LHCB, might be involved in Saccharum narengaleaves in response to water deficit.

In plant, the perception of water deficit can trigger the activation of abscisic acid (ABA)-dependent and ABA-independent regulatory systems that govern drought-inducible gene expression [2]. Genes in response to the water-deficit stress can be divided into two groups based on their functions $[14,15]$. The first group of gene products are involved in the protection of cells and in the regulation of signal transduction pathways of stress responses, such as chaperons, LEA proteins, water channel proteins, heat shock proteins and lipid-transfer proteins. In this study, 90 genes encoding different chaperons have been identified (Additional file 2) in Saccharum narengaleaves. Differential expression analysis identified 14 up-regulated and 7 down-regulated chaperone genes in DTS samples compared to CK (Additional file 4). These chaperones may function in the protection of proteins from degradation and the action of proteinases in sugarcane leaves under drought stress [40]. LEA genes have been reported to be involved in the protection of cell structures from the effects of water loss [41]. The LEA gene products are proposed to be located in cytoplasm and have several features such as hydrophilism, biased in amino acid composition, and lacking in Cys and Trp [41]. They mainly function in sequestration of ions, protection of other proteins or membranes, and renaturation of unfolded proteins [41]. In this study, five LEA genes are identified to be up-regulated in sugarcane leaves in response to water deficit, which is consistent with other plant species like Arabidopsis [42], rice [6], maritime pine [10], loranthus [12] and sugarcane cultivars [11].

Genes encoding heat shock proteins have also been identified to contribute to drought-stress tolerance in plants [2]. In different tissues (root, stem and leaf) of an Indian sugarcane variety (CoS 767), genes encoding heat shock proteins are shown to be up-regulated in response water deficit [18]. In addition, genes encoding ribosomal proteins and putative disease-resistance proteins are also identified to be up-regulated in these sugarcane tissues in response to water deficit [18]. In this study, we confirmed their dysregulation in sugarcane leaves in response to water deficit. Further, we found not only up-regulated but also down-regulated genes that can encode ribosomal proteins and putative disease resistance proteins. For example, genes encoding $30 \mathrm{~S}$ and $50 \mathrm{~S}$ ribosomal proteins were up-regulated while genes encoding $60 \mathrm{~S}$ ribosomal proteins were down-regulated in DTS samples compared to CK (Fig. 5g). DEGs identified in this study include 14 up-regulated and 45 down-regulated genes encoding disease resistance proteins (Additional file 4).

Like ribosomal protein and disease resistance protein, it is difficult to determine the regulation of genes encoding the major water channel proteins - aquaporins - in plant leaves under water-deficit stress. Aquaporins are water transporter proteins that play an important role in adjusting the water status in response to environmental changes [43]. In sugarcane, only TIP aquaporin genes have been identified to be induced by water-deficit stress [17]. Interestingly, our results revealed that the TIP aquaporin genes were down-regulated in Saccharum narengaleaves in response to water deficit while genes encoding NIP and PIP aquaporins were up-regulated. It has been shown that in Arabidopsis the loss of TIP aquaporin leads to cell and plant death [44]. The functions of different aquaporin families in Saccharum narengaleaves in response to water deficit require to be explored with further experiments. Other gene products involved in signal transduction like lipid-transfer proteins and LHCB proteins have also been reported to be induced by the water-deficit stress in commercial sugarcane varieties $[1,17]$.

The second group of gene products in response to water deficit in plants is comprised of regulatory 
proteins that are involved in further regulation of signal transduction and stress-responsive gene expression, such as various TFs and dehydration-responsive element [2]. Rocha and colleagues identified 93 TF genes, including MYB and WRKY TFs, differentially expressed in sugarcane plant (cultivar SP90-1638) in response to water deficit [45]. In our study, ABA-inducible TF genes (e.g., MYB and NAC) and ABA-independent TF genes (e.g., ERF) were identified to be differentially expressed in Saccharum narengaleaves in response to water deficit (Fig. 5d, Additional file 4). In addition, genes encoding DIVARICATA, heat stress, TCP TFs and nuclear transcription factor Y subunit were also identified, for the first time, to be differentially expressed in the leaves in response to water deficit (Fig. 5d, Additional file 4). The up-regulation of heat stress TFs might be responsible for the increase of heat shock proteins in DTS samples. The dysregulation of these TF genes requires further experiments to understand their regulation in Saccharum narengaleaves in response to water deficit. In addition, another group of ABA-independent proteins, dehydration-responsive element-binding proteins, were up-regulated in DTS samples compared to CK, which is consistent with other studies about sugarcane plants under drought stress [11, 17]. Gentile and colleagues reviewed and uncovered a complex regulation of sugarcane miRNAs in response to drought [39]. They point out that the cultivar, the growth conditions and the duration of stress have influence on the observation of miRNA expression profiles. Some miRNAs have been reported to be dysregulated in sugarcane exposed to drought, such as miR160, miR166, miR169, miR171 and miR399 [39]. It has been found in one sugarcane cultivar that the down-regulation of miR169 could increase the expression of glutathione S-transferase (GST) therefore reduce the toxic effects of reactive oxygen species [39]. Although this regulation was not observed in this study, the down-regulation of genes encoding C7254_GLYUR (11-oxo-beta-amyrin 30-oxidase) and ACA6_ORYSJ (Probable calcium-transporting ATPase 6, plasma membrane-type) might be regulated by miR169 in the sugarcane leaves. In addition, we found that some miRNAs could target the DEGs that have been described as being related to drought stress and/or increasing tolerance to water deficit, such as miR164 targeting NAC domain-containing TFs [46]. In addition, we found two miRNAs miR6284 and miR854a of interest because they can regulate multiple gene families, such as NAC domain-containing protein, LEA, ethylene-responsive $\mathrm{TF}$, disease resistance protein, ribosomal protein and WRKY TF. miR854a has been reported to be induced by drought stress in other plants, including rice [47], maize [48], banana [8] and tea [13]. miR6284 has been reported to be an arbuscular mycorrhiza (AM)-responsive miRNA in tomato [49]. The potential regulation of these two miRNAs and their target genes indicates they might be functional in sugarcane leaves in response to water deficit.

Our study agreed the expression changes of some known gene families in Saccharum narenga under water stress, such as LEA, ribosomal protein, heat shock protein, and some TFs. However, it wa shown that aquaporin and DIVARICATA, heat stress, TCP TFs and nuclear transcription factor $Y$ subunit require more experiments to explore their functions in the plants in response to water loss. More importantly, deep sequencing and de novo assembly analysis enabled us to identify new genes in the process. In total, we identified that 3389 genes were differentially expressed in DTS compared to CK, and that 2232 (65.86\%) were similar to known gene families (Additional file 4). Some of them are probably specific to this species or possible noncoding genes (Fig. 2), and some of them were highly expressed (>100 TPM) and dysregulated in DTS, such as TRINITY_DN29490_c3_g1, TRINITY_DN15129_c 0_g1, TRINITY_DN25403_c0_g5 and TRINITY_DN22079 _c0_g3 (Additional file 4). The reason why these possible novel genes were identified require more experiments to explore. Further, their functions in drought tolerance in Saccharum narenga are still unknown and need to be studied.

\section{Conclusions}

In conclusion, we used deep sequencing technology to investigate the transcriptome profiles in Saccharum narenga, a wild type sugarcane leaves in response to water deficit. Among the assembled 104,644 Saccharum narenga genes, 38,721 can be annotated to other databases, such as NR, UniProt, KEGG and GO. In DTS and CK samples we identified 30,297 genes whose expression levels were greater than 5 TPM. Then, edgeR was used for differential expression analysis and 3389 DEGs (1772 up-regulated and 1617 down-regulated) were consistent in paired-end and single-end sequencing. Functional analysis showed they were involved in pathways like "response to blue light", "metabolic pathways" and "plant hormone signal transduction". We observed the expression patterns of several gene families in sugarcane leaves under the water-deficit stress, such as aquaporin, LEA, auxin related proteins, TF, heat shock protein, LHCB, disease resistance protein and ribosomal proteins. Interestingly, genes encoding NIP and PIP aquaporins were up-regulated in Saccharum narenga leaves in response to the water-deficit stress while genes encoding TIP aquaporins were down-regulated. We also found that genes encoding $30 \mathrm{~S}$ and $50 \mathrm{~S}$ ribosomal proteins were up-regulated while $60 \mathrm{~S}$ ribosomal protein genes were down-regulated in DTS samples compared to CK. Further, we showed that the DEGs identified in this study might be regulated by miRNAs. This is the first 
sugarcane leaf transcriptome study using both single-end and paired-end sequencing technologies. Genes encoding LHCB, DIVARICATA and heat stress TFs were first reported, to our knowledge, in sugarcane leaves under water stress. Our findings will improve the understanding of the mechanism gene regulation in sugarcane leaves under drought stress. The output of this study will also contribute to the sugarcane breeding program.

\section{Additional files}

Additional file 1: Gene Ontology annotation for the assembled sugarcane transcriptome. (PDF $192 \mathrm{~kb}$ )

Additional file 2: Gene expression profile in all samples (> 5 TPM) (XLSX $2393 \mathrm{~kb})$

Additional file 3: Differential expression analysis of all genes in DTS samples compared to CK. (XLSX $12539 \mathrm{~kb}$ )

Additional file 4: DEGs consistent in paired-end and single-end sequencing technologies. (XLSX 649 kb)

Additional file 5: $q P C R$ primers. (XLSX $9 \mathrm{~kb})$

Additional file 6: miRNA regulation of the DEGs identified in DTS and CK samples. (XLSX $61 \mathrm{~kb}$ )

\section{Abbreviations}

CK: Control sugarcane leaves; COG: Clusters of Orthologous Groups; DTS: Drought treated sugarcane leaves; ERF: Ethylene-responsive transcription factor; GO: Gene ontology; GST: Glutathione S-transferase; HSP: Heat shock protein; KEGG: Kyoto encyclopedia of genes and genomes; KOG: EuKaryotic Orthologous Group; Log2FC: Log2 fold change; NOG: Nonsupervised orthologous group; NR: NCBI non-redundant database; RSEM: RNA-Seq by Expectation-Maximization; TF: Transcription factor; TPM: Transcripts per million reads

\section{Acknowledgements}

We acknowledge Mr. Dajie Zhou from Beijing Genomics Institute (Shenzhen) for the assistance of transcriptome sequencing.

\section{Funding}

This work was financially supported by the National Science Foundation of China (31101195), Guangxi Special Funds for Scientific Base and Talent (GKAD17195100), Fund of Guangxi Academy of Agricultural Sciences (GNK2018YT02 and GNK2018YM01) and Fund for Guangxi Innovation Teams of Modern Agriculture Technology (gjnytxgxcxtd-03). The Funding bodies had no influence over the experimental design, data analysis or interpretation, or manuscript writing.

\section{Availability of data and materials}

Clean data (FASTQ files) for all samples can be accessed in the NCBI Sequence Read Archive (SRA) database (http://trace.ncbi.nlm.nih.gov/Traces/sra/) under the accession number of SRA633912. This Transcriptome Shotgun Assembly project has been deposited at DDBJ/EMBL/GenBank under the accession GGAS00000000. The version described in this paper is the first version, GGAS01000000.

\section{Authors' contributions}

$\mathrm{XL}, \mathrm{HT}$ and $\mathrm{YL}$ designed the research; $\mathrm{RZ}$ and $\mathrm{HO}$ performed the sample preparation and total RNA extraction; YG, JW and $\mathrm{HZ}$ analyzed the RNA-Seq data; $\mathrm{XL}$ and $\mathrm{HO}$ wrote the manuscript; $\mathrm{HT}$ and $\mathrm{YL}$ revised the manuscript; All authors read and approved the final manuscript.

\section{Ethics approval}

No specific permits were required for the described field studies. The location is not privately-owned or protected in any way, and the field studies did not involve endangered or protected species.
Consent for publication

Not applicable.

\section{Competing interests}

The authors declare that they have no competing interests.

\section{Publisher's Note}

Springer Nature remains neutral with regard to jurisdictional claims in published maps and institutional affiliations.

\section{Author details}

${ }^{1}$ Key Laboratory of Sugarcane Biotechnology and Genetic Improvement (Guangxi), Guangxi Key Laboratory of Sugarcane Genetic Improvement, Ministry of Agriculture, Sugarcane Research Center, Chinese Academy of Agricultural Sciences, Sugarcane Research Institute, Guangxi Academy of Agricultural Sciences, Nanning 530007, China. ${ }^{2}$ State Key Laboratory for Conservation and Utilization of Subtropical Agro-bioresources, Agricultural College, Guangxi University, Nanning 530005, China.

Received: 4 July 2018 Accepted: 16 September 2018

Published online: 20 October 2018

\section{References}

1. Rodrigues FA, de Laia ML, Zingaretti SM. Analysis of gene expression profiles under water stress in tolerant and sensitive sugarcane plants. Plant Sci. 2009;176(2):286-302.

2. Shinozaki K, Yamaguchi-Shinozaki K. Gene networks involved in drought stress response and tolerance. J Exp Bot. 2007:58(2):221-7.

3. Bray EA. Genes commonly regulated by water-deficit stress in Arabidopsis thaliana. J Exp Bot. 2004;55(407):2331-41.

4. Bray EA. Abscisic acid regulation of gene expression during water-deficit stress in the era of the Arabidopsis genome. Plant Cell Environ. 2002;25(2): 153-61.

5. Yu LX, Setter TL. Comparative transcriptional profiling of placenta and endosperm in developing maize kernels in response to water deficit. Plant Physiol. 2003;131(2):568-82.

6. Xu D, Duan X, Wang B, Hong B, Ho T, Wu R. Expression of a late embryogenesis abundant protein gene, HVA1, from barley confers tolerance to water deficit and salt stress in transgenic Rice. Plant Physiol. 1996;110(1): 249-57.

7. Hsieh TH, Lee JT, Charng YY, Chan MT. Tomato plants ectopically expressing Arabidopsis CBF1 show enhanced resistance to water deficit stress. Plant Physiol. 2002;130(2):618-26

8. Muthusamy M, Uma S, Backiyarani S, Saraswathi M. Genome-wide screening for novel, drought stress-responsive long non-coding RNAs in droughtstressed leaf transcriptome of drought-tolerant and-susceptible banana (Musa spp) cultivars using Illumina high-throughput sequencing. Plant Biotechnol Rep. 2015:9(5):279-86.

9. Song L, Prince S, Valliyodan B, Joshi T, Maldonado dos Santos JV, Wang J, Lin L, Wan J, Wang Y, Xu D, et al. Genome-wide transcriptome analysis of soybean primary root under varying water-deficit conditions. BMC Genomics. 2016:17:57.

10. Dubos C, Plomion C. Identification of water-deficit responsive genes in maritime pine (Pinus pinaster Ait.) roots. Plant Mol Biol. 2003;51(2):249-62.

11. Iskandar HM, Casu RE, Fletcher AT, Schmidt S, Xu J, Maclean DJ, Manners $J M$, Bonnett GD. Identification of drought-response genes and a study of their expression during sucrose accumulation and water deficit in sugarcane culms. BMC Plant Biol. 2011;11:12

12. Wei S, Ma X, Pan L, Miao J, Fu J, Bai L, Zhang Z, Guan Y, Mo C, Huang H, et al. Transcriptome Analysis of Taxillusi chinensis (DC.) Danser Seeds in Response to Water Loss. PLoS One. 2017;12(1):e0169177.

13. Guo Y, Zhao S, Zhu C, Chang X, Yue C, Wang Z, Lin Y, Lai Z. Identification of drought-responsive miRNAs and physiological characterization of tea plant (Camellia sinensis L.) under drought stress. BMC Plant Biol. 2017;17(1):211.

14. Yamaguchi-Shinozaki K, Shinozaki K. Transcriptional regulatory networks in cellular responses and tolerance to dehydration and cold stresses. Annu Rev Plant Biol. 2006;57:781-803.

15. Shinozaki K, Yamaguchi-Shinozaki K. Gene expression and signal transduction in water-stress response. Plant Physiol. 1997;115(2):327-34. 
16. Lembke CG, Nishiyama MY Jr, Sato PM, de Andrade RF, Souza GM. Identification of sense and antisense transcripts regulated by drought in sugarcane. Plant Mol Biol. 2012;79(4-5):461-77.

17. Rodrigues F, Da Graça J, De Laia M, Nhani-Jr A, Galbiati J, Ferro M, Ferro J, Zingaretti S. Sugarcane genes differentially expressed during water deficit. Biol Plant. 2011;55(1):43-53.

18. Gupta V, Raghuvanshi S, Gupta A, Saini N, Gaur A, Khan MS, Gupta RS, Singh J, Duttamajumder SK, Srivastava S, et al. The water-deficit stress- and redrot-related genes in sugarcane. Funct Integr Genomics. 2010;10(2):207-14.

19. Prabu G, Kawar P, Pagariya M, Prasad D. Identification of water deficit stress upregulated genes in sugarcane. Plant Mol Biol Rep. 2011;29:291-304.

20. Chen M, Xu R, Ji H, Greening DW, Rai A, Izumikawa K, Ishikawa H, Takahashi N, Simpson RJ. Transcriptome and long noncoding RNA sequencing of three extracellular vesicle subtypes released from the human colon cancer LIM1863 cell line. Sci Rep. 2016:6:38397.

21. Ji H, Chen M, Greening DW, He W, Rai A, Zhang W, Simpson RJ. Deep sequencing of RNA from three different extracellular vesicle (EV) subtypes released from the human LIM1863 colon cancer cell line uncovers distinct miRNA-enrichment signatures. PLoS One. 2014;9(10):e110314.

22. Liu W, Chen M, Bai L, Zhuang Z, Fan C, Jiang N, Zhao J, Ma S, Xiang X. Comprehensive transcriptomics and proteomics analyses of pollinated and parthenocarpic litchi (Litchi chinensis Sonn.) fruits during early development. Sci Rep. 2017;7(1):5401.

23. Haas BJ, Papanicolaou A, Yassour M, Grabherr M, Blood PD, Bowden J, Couger MB, Eccles D, Li B, Lieber $M$, et al. De novo transcript sequence reconstruction from RNA-seq using the trinity platform for reference generation and analysis. Nat Protoc. 2013;8(8):1494-512.

24. Davidson NM, Hawkins ADK, Oshlack A. SuperTranscripts: a data driven reference for analysis and visualisation of transcriptomes. Genome Biol. 2017;18(1):148.

25. Finn RD, Clements J, Eddy SR. HMMER web server: interactive sequence similarity searching. Nucleic Acids Res. 2011;39(Web Server issue):W29-37.

26. Petersen TN, Brunak S, von Heijne G, Nielsen H. SignalP 4.0: discriminating signal peptides from transmembrane regions. Nat Methods. 2011;8(10):785-6.

27. Lagesen K, Hallin P, Rodland EA, Staerfeldt HH, Rognes T, Ussery DW. RNAmmer: consistent and rapid annotation of ribosomal RNA genes. Nucleic Acids Res. 2007:35(9):3100-8.

28. Krogh A, Larsson B, von Heijne G, Sonnhammer EL. Predicting transmembrane protein topology with a hidden Markov model: application to complete genomes. J Mol Biol. 2001;305(3):567-80.

29. Powell S, Forslund K, Szklarczyk D, Trachana K, Roth A, Huerta-Cepas J, Gabaldon T, Rattei T, Creevey C, Kuhn M, et al. eggNOG v4.0: nested orthology inference across 3686 organisms. Nucleic Acids Res. 2014; 42(Database issue):D231-9.

30. Altschul SF, Madden TL, Schaffer AA, Zhang J, Zhang Z, Miller W, Lipman DJ. Gapped BLAST and PSI-BLAST: a new generation of protein database search programs. Nucleic Acids Res. 1997;25(17):3389-402.

31. Li B, Ruotti V, Stewart RM, Thomson JA, Dewey CN. RNA-Seq gene expression estimation with read mapping uncertainty. Bioinformatics. 2010; 26(4):493-500

32. Langmead B, Salzberg SL. Fast gapped-read alignment with bowtie 2. Nat Methods. 2012:9(4):357-9.

33. Robinson MD, McCarthy DJ, Smyth GK. edgeR: a Bioconductor package for differential expression analysis of digital gene expression data. Bioinformatics. 2010;26(1):139-40.

34. Wu HJ, Ma YK, Chen T, Wang M, Wang XJ. PsRobot: a web-based plant small RNA meta-analysis toolbox. Nucleic Acids Res. 2012;40(Web Server issue):W22-8.

35. Vacca RA, de Pinto MC, Valenti D, Passarella S, Marra E, De Gara L. Production of reactive oxygen species, alteration of cytosolic ascorbate peroxidase, and impairment of mitochondrial metabolism are early events in heat shock-induced programmed cell death in tobacco bright-yellow 2 cells. Plant Physiol. 2004;134(3):1100-12.

36. Wu HM, Cheun AY. Programmed cell death in plant reproduction. Plant Mol Biol. 2000:44(3):267-81

37. Jones AM. Programmed cell death in development and defense. Plant Physiol. 2001;125(1):94-7.

38. Li M, Liang Z, He S, Zeng Y, Jing Y, Fang W, Wu K, Wang G, Ning X, Wang L, et al. Genome-wide identification of leaf abscission associated microRNAs in sugarcane (Saccharum officinarum L.). BMC Genomics. 2017;18(1):754.
39. Gentile A, Dias LI, Mattos RS, Ferreira TH, Menossi M. MicroRNAs and drought responses in sugarcane. Front Plant Sci. 2015;6:58.

40. Mahajan S, Tuteja N. Cold, salinity and drought stresses: an overview. Arch Biochem Biophys. 2005:444(2):139-58.

41. Bray EA. Molecular responses to water deficit. Plant Physiol. 1993;103(4): 1035-40.

42. Olvera-Carrillo Y, Campos F, Reyes JL, Garciarrubio A, Covarrubias AA. Functional analysis of the group 4 late embryogenesis abundant proteins reveals their relevance in the adaptive response during water deficit in Arabidopsis. Plant Physiol. 2010;154(1):373-90.

43. LUU DT, Maurel C. Aquaporins in a challenging environment: molecular gears for adjusting plant water status. Plant Cell Environ. 2005;28(1):85-96.

44. Ma S, Quist TM, Ulanov A, Joly R, Bohnert HJ. Loss of TIP1;1 aquaporin in Arabidopsis leads to cell and plant death. Plant J. 2004;40(6):845-59.

45. Rocha FR, Papini-Terzi FS, Nishiyama MY Jr, Vencio RZ, Vicentini R, Duarte RD, de Rosa VE Jr, Vinagre F, Barsalobres C, Medeiros AH, et al. Signal transduction-related responses to phytohormones and environmental challenges in sugarcane. BMC Genomics. 2007:8:71.

46. Guo HS, Xie Q, Fei JF, Chua NH. MicroRNA directs mRNA cleavage of the transcription factor NAC1 to downregulate auxin signals for arabidopsis lateral root development. Plant Cell. 2005;17(5):1376-86.

47. Zhou L, Liu Y, Liu Z, Kong D, Duan M, Luo L. Genome-wide identification and analysis of drought-responsive microRNAs in Oryza sativa. J Exp Bot. 2010;61(15):4157-68.

48. Wang YG, An M, Zhou SF, She YH, Li WC, Fu FL. Expression profile of maize microRNAs corresponding to their target genes under drought stress. Biochem Genet. 2014;52(11-12):474-93.

49. Wu P, Wu Y, Liu CC, Liu LW, Ma FF, Wu XY, Wu M, Hang YY, Chen JQ, Shao $Z Q$, et al. Identification of arbuscular mycorrhiza (AM)-responsive microRNAs in tomato. Front Plant Sci. 2016;7:429.

Ready to submit your research? Choose BMC and benefit from

- fast, convenient online submission

- thorough peer review by experienced researchers in your field

- rapid publication on acceptance

- support for research data, including large and complex data types

- gold Open Access which fosters wider collaboration and increased citations

- maximum visibility for your research: over $100 \mathrm{M}$ website views per year

At $\mathrm{BMC}$, research is always in progress.

Learn more biomedcentral.com/submissions 